\title{
VLF Propagation in a Compressible Ionosphere
}

\author{
Richard B. Kieburtz \\ Contribution From New York University School of Engineering and Science, Bronx, N.Y.
}

(Received January 28, 1964)

\begin{abstract}
The effect of finite compressibility of the electron gas is taken into account in the calculation of the propagation constant and the power carried by a VLF wave propagating in the ionosphere.

The principal result is that the electromagnetic wave ("whistler" mode) and a quasi-acoustic or compressional wave are coupled near the critical, or maximum, angle of the wave normal with respect to the direction of the geomagnetic field. Near the critical angle, the direction of power flow is no longer constrained to lie within a narrow cone of angles about the geomagnetic field, but energy can propagate in a quasi-acoustic mode at all angles up to the critical angle of the wave normal. Because of collisional damping, the effects of finite compressibility do not appear to be detectable unless the electron temperature in the outer ionosphere exceeds $10^{4} \mathrm{~K}$.
\end{abstract}

\section{Introduction}

Recent experiments in communication with satellites and space probes have stimulated considerable interest in studies of propagation of electromagnetic waves in ionized gases. This interest has not been confined to the higher radiofrequencies, but extends to the VLF band as well. Although much of the basic phenomenon of VLF propagation within the ionosphere has been satisfactorily explained by Storey [1953] and by more recent workers (for example, [Helliwell and Morgan, 1959; Smith, Helliwell, and Yabroff, 1960; Yabroff, 1961]), in connection with the study of atmospheric "whistlers," the possibility of performing satellite experiments [Leiphart et al., 1962; Cain et al., 1961] at VLF, utilizing antennas located within the ionosphere, affords the opportunity of measuring other effects which may not be measured directly from the ground.

One of the effects which has not previously been accounted for in the theory of ionospheric propagation is the finite compressibility of the electron gas. The effects of finite compressibility may not be apparent at frequencies above the gyrofrequency, because of the great discrepancy in the propagation velocities of electromagnetic and acoustic waves. At VLF, however, the great phase retardation of the electromagnetic wave which can occur appears to provide favorable conditions for interaction of the electromagnetic wave with an acoustic, or compressional wave. It is the purpose of this paper to investigate the effects of finite compressibility on the propagation of small-signal, plane waves at VLF frequencies in a uniform ionosphere.

\section{Magneto-ionic Theory for an Electron Gas}

In considering propagation at frequencies of several kilohertz and higher, the motions of heavy ions may be ignored, since the ion plasma frequency and ionic gyrofrequency in the earth's atmosphere are less than a few hundred hertz.

The field equations for a time-harmonic $\left(e^{j \omega t}\right)$ electromagnetic field in an electron gas can then be written [Oster, 1960] in linearized form for fields of small amplitude $\left(\mu_{0} H_{r f}<<B_{0}\right)$, and for zero average drift velocity of the electron gas,

$$
\begin{gathered}
\nabla \times \mathbf{E}=-j \omega \mu_{0} \mathbf{H} \\
\nabla \times \mathbf{H}=j \omega \epsilon_{0} \mathbf{E}-N e \mathbf{v}
\end{gathered}
$$

${ }^{1}$ Part of the results presented in this paper were given in a paper presented at the IE EE Symposium on Antennas and Propagation in Boulder, Colo., July 1963. 


$$
\begin{gathered}
\nabla \cdot \mathbf{v}=-\frac{j \omega}{\gamma P_{0}} p \\
\nabla p=-(j \omega+\nu) m N \mathbf{v}-N e\left(\mathbf{E}+\mathbf{v} \times \mathbf{B}_{0}\right)
\end{gathered}
$$

where

$\mathbf{E}, \mathbf{H}$ represent the time-varying electromagnetic field intensities,

$\mathbf{B}_{0}$ is the flux density of the geomagnetic field,

$N$ is the average electron density,

$\mathbf{v}$ is the electron velocity, averaged over a small element of volume whose radius is the Debye length,

$e$ is electronic charge,

$m$ is electron mass,

$\nu$ is the electron collision frequency,

$P_{0}$ is the time-averaged pressure of the electron gas,

$p$ is the time-varying component of pressure,

$\gamma$ is the ratio of specific heats, equal to $5 / 3$ for a gas of free electrons.

Equations (1) and (2) are just Maxwell's equations, taking into account convection currents due to electron motion; (3) is the combined equation of continuity and state, and (4) is the momentum equation.

Equations (3) and (4) can be combined to give

$$
-\frac{\gamma P_{0}}{j \omega} \nabla \nabla \cdot \mathbf{v}=-(j \omega+\nu) m N \mathbf{v}-N e\left(\mathbf{E}+\mathbf{v} \times \mathbf{B}_{0}\right)
$$

which now gives the behavior of the electron plasma, expressed in terms of the velocity, in response to the electromagnetic forces acting upon it.

Now to solve the field equations (1), (2), and (5), consider propagation of a plane wave. Expressing the field vectors in terms of a Cartesian coordinate system, and taking the z-axis along the direction of propagation, it is seen that the spatial dependence of all time-varying quantities is given by $e^{-j \beta z}$, where $\beta$ is the propagation constant, which may be complex.

The two Maxwell equations, (1) and (2), may be combined to give a wave equation,

$$
\nabla \times \nabla \times \mathbf{E}-k_{0}^{2} \mathbf{E}=j \omega \mu_{0} N e \mathbf{v}
$$

where $k_{0}=\omega \sqrt{\mu_{0} \epsilon_{0}}$ is the wave number in free space. In view of the assumed plane-wave spatial dependence of the fields, (6) becomes

$$
\left(k_{0}^{2}-\beta^{2}-\nabla \nabla \cdot\right) \mathbf{E}=-j \omega \mu_{0} N e \mathbf{v} .
$$

It is convenient to write $(7)$ in matrix form, observing that $(\nabla \cdot \mathbf{E})=\beta^{2} E_{z}$, obtaining

$$
j \frac{e}{\omega m}\left[\begin{array}{ccr}
k_{0}^{2}-\beta^{2} & 0 & 0 \\
0 & k_{0}^{2}-\beta^{2} & 0 \\
0 & 0 & k_{0}^{2}
\end{array}\right] . \mathbf{E}=k_{n}^{2} \mathbf{v}
$$

where $\mathrm{k}_{n}^{2}=\frac{\mu_{0} N e^{2}}{m}=\omega_{n}^{2} \mu_{0} \epsilon_{0}$ is the square of the wave number corresponding to the electron plasma frequency. Similarly, by taking the coordinate system such that $B_{0}$ lies in the $x-z$ 
plane, (5) can be written as

$$
\begin{aligned}
\mathbf{E} & =-j \frac{\omega m}{e}\left[\begin{array}{ccc}
1-j \frac{\nu}{\omega} & -j \frac{\omega_{L}}{\omega} & 0 \\
j \frac{\omega_{L}}{\omega} & 1-j \frac{\nu}{\omega} & -j \frac{\omega_{T}}{\omega} \\
0 & j \frac{\omega_{T}}{\omega} & 1-j \frac{\nu}{\omega}-\frac{\mu^{2} \beta^{2}}{\omega^{2}}
\end{array}\right] \cdot \mathbf{v} \\
& =-j \frac{\omega m}{e} L \cdot \mathbf{v}
\end{aligned}
$$

where

$u^{2}=\frac{\gamma P_{0}}{N m}$ is the square of the acoustic velocity in the medium,

$\omega_{L}=\omega \cos \theta$,

$\omega_{T}=\omega_{0} \sin \theta$,

$\omega_{0}=\frac{e}{m} B_{0}$, the electron radian gyrofrequency,

$\theta$ is the angle between the direction of the wave normal and the geomagnetic field.

The acoustic velocity is directly related to the temperature of the electron gas, $u^{2}=\frac{\gamma k T}{m}$, where $T$ is in degrees Kelvin, and $k$ is the Boltzmann constant.

Combining (8) and (9) and eliminating $\mathbf{E}$ gives a determinantal equation for the propagation constant,

$$
\operatorname{det}\left[\begin{array}{ccc}
\left(1-j \frac{\nu}{\omega}\right)\left(k_{0}^{2}-\beta^{2}\right)-k_{n}^{2} & -j \frac{\omega_{L}}{\omega}\left(k_{0}^{2}-\beta^{2}\right) & 0 \\
j \frac{\omega_{L}}{\omega}\left(k_{0}^{2}-\beta^{2}\right) & \left(1-j \frac{\nu}{\omega}\right)\left(k_{0}^{2}-\beta^{2}\right)-k_{n}^{2} & -j \frac{\omega_{T}}{\omega}\left(k_{0}^{2}-\beta^{2}\right) \\
0 & j \frac{\omega_{T}}{\omega} k_{0}^{2} & \left(1-j \frac{\nu}{\omega}\right) k_{0}^{2}-\frac{u^{2} \beta^{2}}{\omega^{2}}-k_{n}^{2}
\end{array}\right]=0 .
$$

It is the solution of (10) for values of $\beta$ which is now of concern.

\section{Periurbation Solution of the Determinantal Equation}

The determinantal (10) yields a cubic equation in $\beta^{2}$, with complex coefficients. An analytic solution of the equation is difficult except for some special cases, when the direction of propagation is either along, or transverse to the direction of the geomagnetic field. For the transverse case, there are no solutions which yield $\operatorname{Re}\left(\beta^{2}\right)>0$, or a propagating wave. In the longitudinal case, two such solutions occur. One of these values of $\beta^{2}$ corresponds to whistler mode propagation, and the other to a longitudinal acoustic wave.

A perturbation solution to the determinantal equation may be obtained using the solutions for longitudinal propagation as the zero-order solution. The first perturbation of the equation will be shown to give a propagation constant for the electromagnetic wave which is recognizable as the quasi-longitudinal approximation of magneto-ionic theory for an incompressible plasma, 
plus another propagation constant for an acoustic wave. Application of the perturbation method to obtain a second order correction is not fruitful, since the perturbation parameter is not small for all angles $\theta$.

It is useful to rewrite (10) as an operator equation

$$
S_{\lambda} \cdot \mathbf{v}=\left[\begin{array}{ccc}
(1-j Z)(1-\lambda)-X & -j Y_{L}(1-\lambda) & 0 \\
j Y_{L}(1-\lambda) & (1-j Z)(1-\lambda)-X & -j Y_{T}(1-\lambda) \\
0 & j Y_{T} & 1-j Z-V \lambda-X
\end{array}\right] \cdot \mathbf{v}=0
$$

where, in the usual notation for the magneto-ionic theory,

$$
\begin{aligned}
X & =\frac{\omega_{n}^{2}}{\omega^{2}}=\frac{k_{n}^{2}}{k_{0}^{2}}, \\
Y_{L} & =\frac{\omega_{L}}{\omega}, \\
Y_{T} & =\frac{\omega_{T}}{\omega}, \\
Z & =\frac{\nu}{\omega},
\end{aligned}
$$

and, in addition,

$$
V=\frac{u^{2}}{c^{2}}
$$

and $\lambda$ is the square of the refractive index. It can be seen that $\lambda$ is an eigenvalue of an operator $S_{0}$ by separating the terms containing $\lambda$ from $S_{\lambda}$,

$$
S_{\lambda} \cdot \mathbf{v}=\left[S_{0}-\lambda \mu\right] \cdot \mathbf{v}=0 .
$$

The operators $S_{0}$ and $\mu$ are, in matrix form,

where $P=1-j Z$.

$$
\begin{aligned}
S_{0} & =\left[\begin{array}{ccc}
P-X & -j Y_{L} & 0 \\
j Y_{L} & P-X & -j Y_{T} \\
0 & j Y_{T} & P-X
\end{array}\right] \\
\mu & =\left[\begin{array}{ccc}
P & -j Y_{L} & 0 \\
j Y_{L} & P & -j Y_{T} \\
0 & 0 & V
\end{array}\right]
\end{aligned}
$$

The eigenvalue equation (12) can be put into a more conventional form by a coordinate transformation diagonalizing $\mu$. This can be accomplished by defining

which gives the eigenvalue equation

$$
\mathbf{x}=\mu \cdot \mathbf{v},
$$

$$
T \cdot \mathbf{x}-\lambda \mathbf{x}=0
$$

where

$$
T=S_{0} \cdot \mu^{-1}=\left[\begin{array}{ccc}
1-\frac{P X}{P^{2}-Y_{L}^{2}} & -j \frac{X Y_{L}}{P^{2}-Y_{L}^{2}} & \frac{X Y_{L} Y_{T}}{V\left(P^{2}-Y_{L}^{2}\right)} \\
j \frac{X Y_{L}}{P^{2}-Y_{L}^{2}} & 1-\frac{P X}{P^{2}-Y_{L}^{2}} & -j \frac{P X Y_{T}}{V\left(P^{2}-Y_{L}^{2}\right)} \\
\frac{Y_{L} Y_{T}}{P^{2}-Y_{L}^{2}} & j \frac{P Y_{T}}{P^{2}-Y_{L}^{2}} & \frac{P-X}{V}-\frac{P Y_{T}^{2}}{V\left(P^{2}-Y_{L}^{2}\right)}
\end{array}\right]
$$


The operator $T$ is now written as the sum of a zero-order term and a perturbation term,

where

$$
T=T_{0}+T_{1}
$$

$$
T_{0}=\left[\begin{array}{ccc}
1-\frac{P X}{P^{2}-Y_{L}^{2}} & -j \frac{X Y_{L}}{P^{2}-Y_{L}^{2}} & 0 \\
j \frac{X Y_{L}}{P^{2}-Y_{L}^{2}} & 1-\frac{P X}{P^{2}-Y_{L}^{2}} & 0 \\
0 & 0 & \frac{P-X}{V}
\end{array}\right]
$$

and

$$
T_{1}=\frac{Y_{T}}{P^{2}-Y_{L}^{2}}\left[\begin{array}{ccc}
0 & 0 & \frac{X Y_{L}}{V} \\
0 & 0 & -j \frac{P X}{V} \\
Y_{L} & j P & -\frac{P Y_{T}}{V}
\end{array}\right] .
$$

Thus $T_{0}$ corresponds to the quasi-longitudinal approximation of magneto-ionic theory, and the expansion parameter of the perturbation is $Y_{T} / \sqrt{Y_{L}^{2}-P^{2}}$.

The eigenvalues and corresponding normalized eigenvectors of $T_{0}$ are

$$
\begin{array}{llll}
\lambda_{1}^{(0)}=1-\frac{X}{P-Y_{L}}, & \mathbf{x}_{1}^{(0)}=\left(\frac{1}{\sqrt{2}},\right. & \frac{-j}{\sqrt{2}}, & 0), \\
\lambda_{2}^{(0)}=1-\frac{X}{P+Y_{L}}, & \mathbf{x}_{2}^{(0)}=\left(\frac{1}{\sqrt{2}},\right. & \frac{j}{\sqrt{2}}, & 0), \\
\lambda_{3}^{(0)}=\frac{P-X}{V}, & \mathbf{x}_{3}^{(0)}=(0, & 0, & 1) .
\end{array}
$$

In calculating the eigenvectors, the damping has been neglected, by taking $P=1$. This is justifiable for the case of small losses since the eigenvectors are to be used only in power calculations, in which a vector is multiplied by its complex conjugate in order to find real-valued quantities. In such calculations, the error incurred in neglecting $Z$ in the eigenvectors will be of order $Z^{2}$ in the power calculations, and can therefore be neglected for $Z^{2}<<1$.

The first-order approximation to the eigenvalues is now calculated from the zero-order eigenvectors and the operator $T$,

These are

$$
\lambda_{i}^{(1)}=\frac{\overline{\mathbf{x}_{i}^{(0)}} \cdot T \cdot \mathbf{x}_{i}^{(0)}}{\overline{\mathbf{x}_{i}^{(0)}} \cdot \mathbf{x}_{i}^{(0)}} .
$$

$$
\begin{gathered}
\lambda_{1}^{(1)}=1-\frac{X}{P-Y_{L}} \\
\lambda_{2}^{(1)}=1-\frac{X}{P+Y_{L}} \\
\lambda_{3}^{(1)}=\frac{P-X}{V}-\frac{P Y_{T}^{2}}{V\left(P^{2}-Y_{L}^{2}\right)} .
\end{gathered}
$$

Since $V$ is ordinarily a very small number, only terms containing negative powers of $V$ have been retained in the expression for $\lambda_{3}^{(1)}$. 
For $Z<<1$, the complex eigenvalues can be expanded to first power in $Z$, giving

$$
\begin{aligned}
\lambda_{1}^{(1)} & =1-\frac{X}{1-Y_{L}}\left(1+j \frac{Z}{1-Y_{L}}\right), \\
\lambda_{2}^{(1)} & =1-\frac{X}{1+Y_{L}}\left(1+j \frac{Z}{1+Y_{L}}\right), \\
\lambda_{3}^{(1)} & =\frac{1-X-j Z}{V}-\frac{Y_{T}^{2}}{V\left(1-Y_{L}^{2}\right)}\left(1+j \frac{Z}{1-Y_{L}}\right) .
\end{aligned}
$$

It can be seen that the first two eigenvalues still agree with the results of the $Q L$ approximation of magneto-ionic theory, in which only one of these eigenvalues has a positive real part for $X>Y_{L}>1$. The third eigenvalue has a positive real part for $Y_{T}^{2} /\left(Y_{L}^{2}-1\right)>X-1$, which indicates that a quasi-acoustic mode can propagate over a narrow cone of wave-normal angles near the critical angle for whistler mode propagation, which is given approximately by

$$
\theta_{c} \simeq \arccos \frac{1}{Y} \text {. }
$$

The damping of either the electromagnetic whistler mode or of the quasi-acoustic mode becomes large near the critical angle, thus the acoustic mode may not be observed due to excessive damping if the cone of the angles along which it may propagate is too small. The width of this cone can be shown to be approximately given by

$$
\cos 2 \theta_{\mathrm{min}}-\cos 2 \theta_{\max } \simeq \frac{2}{X} .
$$

Thus the acoustic wave with low damping can probably be observed only in the outer ionosphere, above the height of maximum electron density in the $F 2$ layer.

It is now of interest to examine the first-order approximation to the eigenvectors. These are found from perturbation theory by writing the eigenvalue equation as

$$
[T-\lambda] \cdot \mathbf{x}=\left[T_{0}-\lambda^{(0)}\right] \cdot \mathbf{x}^{(0)}+\delta\left[T_{0}-\lambda^{(0)}\right] \cdot\left(\mathbf{x}^{(1)}-\mathbf{x}^{(0)}\right)+\delta\left[T_{1}-\left(\lambda^{(1)}-\lambda^{(0)}\right)\right] \cdot \mathbf{x}^{(0)}+0\left(\delta^{2}\right),
$$

where $\delta$ is a dummy perturbation parameter. Since $\left[T_{0}-\lambda^{(0)}\right] \cdot \mathbf{x}^{(0)}=0, \mathbf{x}^{(1)}$ can be found by setting

$$
\left[T_{0}-\lambda^{(0)}\right] \cdot\left(\mathbf{x}^{(1)}-\mathbf{x}^{(0)}\right)=-\left[T_{1}-\left(\lambda^{(1)}-\lambda^{(0)}\right)\right] \cdot \mathbf{x}^{(0)}
$$

and solving for the vectors $\mathbf{x}^{(1)}$ subject to the restriction

$$
\left(\mathbf{x}^{(1)}-\mathbf{x}^{(0)}\right) \cdot \mathbf{x}^{(0)}=0 .
$$

This restriction enables $\mathbf{x}^{(1)}$ to be specified uniquely. The new set of vectors which are found by this procedure are, again neglecting $Z$ in solving for the eigenvectors,

$$
\begin{aligned}
\mathbf{x}_{1}^{(1)} & =\left(1,-j, \frac{Y_{T} V}{(1-V)\left(1-Y_{L}-X\right)+X Y_{L}}\right), \\
\mathbf{x}_{2}^{(1)} & =\left(1, j,-\frac{Y_{T} V}{(1-V)\left(1+Y_{L}-X\right)-X Y_{L}}\right), \\
\mathbf{x}_{3}^{(1)} & =\left(-\frac{X Y_{T}}{(1-X)\left(1-Y_{L}^{2}\right)}, j \frac{X Y_{T}}{(1-X)\left(1-Y_{L}^{2}\right)}, 1\right) .
\end{aligned}
$$

\section{Poynting Vector}

In order to find the ray direction, or direction of energy transport, it is necessary to evaluate the power flow from the generalized Poynting vector. Thus for a wave having both electro- 
magnetic and acoustic energy, the power density is given by

$$
\mathbf{P}=\frac{1}{2} \operatorname{Re}\left[\mathbf{E} \times \overline{\mathbf{H}}+p \overline{\mathbf{v}}_{\text {a coustic }}\right] .
$$

For plane waves with the wave normal along the $z$ coordinate, this expression can be written as

$$
\mathbf{P}=\frac{1}{2} \operatorname{Re}\left\{\frac{\beta}{\omega \mu_{0}}\left[\mathbf{E} \cdot \overline{\mathbf{E}} \mathbf{a}_{z}-E_{z} \overline{\mathbf{E}}+\left(\frac{\omega m}{e}\right)^{2} X V v_{z} \bar{v}_{z} \mathbf{a}_{z}\right]\right\}
$$

To find the quantities of interest, $\mathbf{v}$ and $\mathbf{E}$ from $\mathbf{x}$, it is necessary to invert the transformation (15), finding

$$
\mathbf{v}=\mu^{-1} \cdot \mathbf{x}
$$

This form is appropriate to find $v_{z}$ for use in the power calculation (30), but is not appropriate to find $\mathbf{E}$. The electromagnetic energy term should not depend on the longitudinal velocity component, so we find that part of the electric field which contributes to the electromagnetic power flow by employing a projection operator,

$$
Q=\left[\begin{array}{lll}
1 & 0 & 0 \\
0 & 1 & 0 \\
0 & 0 & 0
\end{array}\right] .
$$

Then, for use in the calculation of (30),

$$
\mathbf{E}=-j \frac{\omega m}{e} L \cdot \mu^{-1} \cdot Q \cdot \mathbf{x}
$$

where $L$ is the operator defined by $(9)$.

One finds then that

$$
\begin{aligned}
& \mathbf{E}_{1}^{(1)}=-j \frac{\omega m}{e}\left(1,-j, Y_{T} /\left(1-Y_{L}\right)\right), \\
& v_{1 z}^{(1)}=Y_{T} /\left(X\left(V-1+Y_{L}\right)\right),
\end{aligned}
$$

and, for $X<<1$ so that $X /(X-1) \simeq 1$,

$$
\begin{aligned}
\mathbf{E}_{3}^{(1)} & =j \frac{\omega m}{e} \frac{Y_{T}}{1-Y_{L}^{2}}\left(1,-j, Y_{T} /\left(1-Y_{L}\right)\right), \\
V_{3 z}^{(1)} & =1 / V .
\end{aligned}
$$

From these vectors, the power expressions are easily calculated,

$$
\begin{aligned}
& \mathbf{P}_{1}=\frac{\beta_{1}}{\omega \mu_{0}}\left(\frac{\omega m}{e}\right)^{2}\left[\left(2+\frac{V Y_{T}^{2}}{X\left(V-1+Y_{L}\right)^{2}}\right) \mathbf{a}_{z}-\left(\frac{Y_{T}}{1-Y_{L}}\right) \mathbf{a}_{x}\right], \\
& \mathbf{P}_{3}=\frac{\beta_{3}}{\omega \mu_{0}}\left(\frac{\omega m}{e}\right)^{2}\left[\left(2+\frac{X\left(1-Y_{L}^{2}\right)^{2}}{V Y_{T}}\right) \mathbf{a}_{z}-\left(\frac{Y_{T}}{1-Y_{L}}\right) \mathbf{a}_{x}\right] .
\end{aligned}
$$

The ray directions, or directions of power flow, deviate from the wave-normal directions by angles

$$
\psi_{i}=\arctan \left(\frac{P_{i x}}{P_{i z}}\right), \quad i=1,3
$$

Because the ratio $X / V$ is a very large number, it is seen that the ray direction of the quasi- 
acoustic wave, $\mathbf{P}_{3}$, is nearly along the wave normal, whereas that of the quasi-electromagnetic wave, $\mathbf{P}_{1}$, becomes very nearly in the direction of the geomagnetic field for angles near the critical angle, if $Y>>1$.

The wave impedance of the quasi-electromagnetic and the acoustic waves can also be determined from the power calculation. Defining a wave impedance in the direction of the wave normal by

$$
Z_{z}=\frac{|\mathbf{P}|}{H^{2}}
$$

the impedance is found to be

$$
Z_{z}=\frac{\omega \mu_{0}}{\beta}\left[1+\frac{\left(\frac{\omega m}{e}\right)^{2} X V v_{z} \bar{v}_{z}}{\mathbf{E} \cdot \overline{\mathbf{E}}-E_{z} \bar{E}_{z}}\right] .
$$

In the quasi-electromagnetic wave, the ratio of acoustic to electromagnetic energy is small, and the impedance becomes approximately

$$
Z_{1 z} \simeq \frac{\omega \mu_{0}}{\beta_{1}}
$$

For the quasi-acoustic wave, the acoustic energy is many times greater than the electromagnetic energy, and the wave impedance is correspondingly greater,

$$
Z_{3 z} \simeq \frac{\omega \mu_{0}}{\beta_{3}}\left(\frac{\omega m}{e}\right)^{2} X V \frac{v_{z} \overrightarrow{v_{z}}}{\mathbf{E}_{3} \cdot \overline{\mathbf{E}}_{3}-E_{3 z} \bar{E}_{3 z}} .
$$

As a result of the disparity in wave impedances, an antenna which is designed to radiate an electromagnetic wave efficiently will not couple greatly to the quasi-acoustic wave, and in order to observe acoustic wave propagation in the ionosphere, it may be necessary to employ special antennas.

\section{Some Numerical Results}

In figures 1 and 2 the refractive index is given for a lossless ionosphere $(Z=0)$ as a function of wave-normal angle for parameters $X=50, Y=7.8, V=0.002^{2}$, as obtained by a numerical solution of the exact determinantal (10). The refractive indices obtained by means of the first-order perturbation solution are also given in figure 2 for comparison. The longitudinal

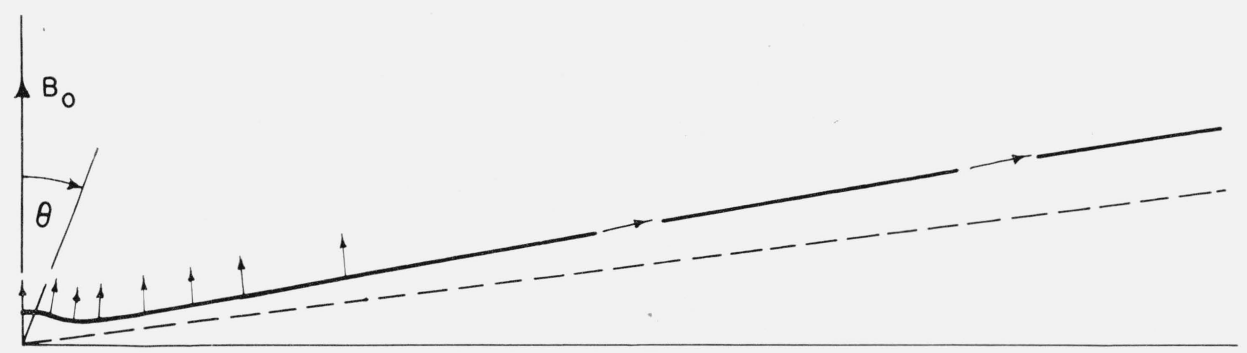

Figure 1. Section of refractive index surface for $\mathrm{X}=50, \mathrm{Y}=7.8, \mathrm{~V}=0.002$.

The angle $\theta$ is the inclination of the wave normal with respect to the geomagnetic field. The arrows along the curve indicate directions of power flow for waves having various wave-normal angles $\theta$.

2 These parameter values correspond roughly to a model of the earth's ionosphere at a distance of slightly more than one earth radius above the earth's surface. The value of $V$ corresponds to an electron temperature of about $7 \times 10^{6}{ }^{\circ} \mathrm{K}$. These values were not chosen to give a realistic approximation to any actual conditions so much as they were chosen to give results which could be illustrated graphically. A more realistic set of values would have $\mathrm{X} \sim 400-7000$ and $V \sim 5 \times\left(10^{-5}-10^{-7}\right)$, yielding a larger critical angle and a narrower cone of angles in which the quasiacoustic wave can propagate. 


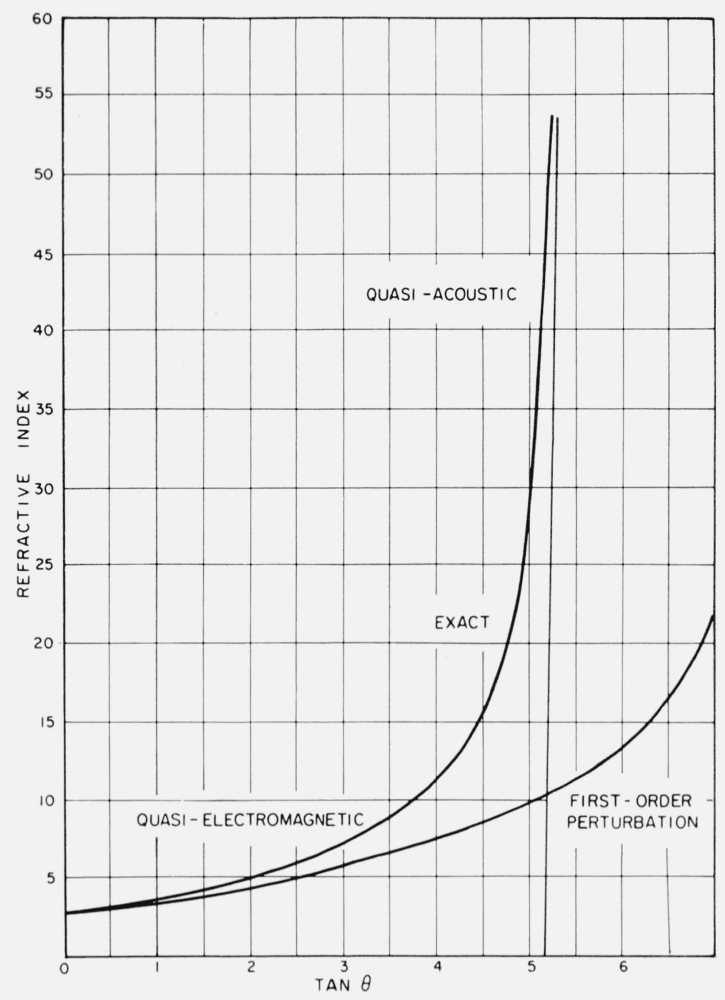

Figure 2. Refractive index as a function of $\tan \theta$, indicating the transition from a quasi-electromagnetic to a quasi-acoustic wave.

The first-order perturbation solution is compared with the exact solution of the determinental equation for $X=50, Y=7.8, V=0.002$.

acoustic wave is not shown in these figures. It is confined to wave-normal angles very near the direction of the geomagnetic field and does not couple with the electromagnetic wave.

The curve of figure 1 shows the refractive index and the direction of power flow, or ray direction, versus wave-normal angle with respect to the direction of the geomagnetic field. Up to the angle of transition from the quasi-electromagnetic to the quasi-acoustic wave, the refractive index is similar to that for the whistler mode in a cold or incompressible ionosphere, and the ray direction is approximately normal to the plane tangent to the refractive index surface, as for the incompressible case. For larger angles, the wave becomes primarily acoustic in nature; the refractive index increases very rapidly with the wave normal angle, and the ray direction is nearly along the wave normal.

From the approximate form (24c) for the square of refractive index, it is seen that the quasi-acoustic wave exists from an angle $\theta_{A}$ to $\theta_{c}$, where the angles are related by

$$
\cos ^{2} \theta_{A}-\cos ^{2} \theta_{c}=\frac{Y^{2}-1}{Y^{2} X} \simeq \frac{1}{X}
$$

\section{Damping Effects}

Damping effects can be accounted for by collisions between electrons and heavy particles, principally positive ions and neutral atoms. The scattering cross section presented by charged ions to electrons is relatively very large at low electron temperatures, due to the long range of the Coulomb force. However, as the temperature, or mean kinetic energy of electrons increases, the scattering cross section of ions decreases, until at approximately $1.8 \times 10^{5}{ }^{\circ} \mathrm{K}$ the scattering 
cross section presented by the Coulomb potential is no greater than is that of short range forces. The result is that the collision frequency in the ionosphere can be extremely low if the electron temperature is of the order of $10^{5}{ }^{\circ} \mathrm{K}$. For instance, if $X=50, T=10^{5}{ }^{\circ} \mathrm{K}$, and it is assumed that the density of positive ions is equal to that of electrons, while the density of all heavy particles is 10 times that of electrons, then the collision frequency, estimated by the formulas given by Cowling [1945] would be approximately $8 \times 10^{-4}$, giving $Z$ at $18 \mathrm{kHz}$ equal to $4.4 \times 10^{-8}$.

The damping of the quasi-acoustic wave will be greater than that of the quasi-electromagnetic wave, particularly near the region of coupling of the two waves. The ratio of attenuation constant to propagation constant for each of the waves is approximately

$$
\begin{gathered}
\tan \phi_{1}=\frac{\left|\operatorname{Im} \beta_{1}^{(1)}\right|}{\operatorname{Re} \beta_{1}^{(1)}} \simeq \frac{1}{2} \frac{Z}{Y_{L}-1}, \\
\tan \phi_{3}=\frac{\left|\operatorname{Im} \beta_{3}^{(1)}\right|}{\operatorname{Re} \beta_{3}^{(1)}} \simeq \frac{1}{2} \frac{\mathrm{Z}}{\left(Y_{L}-1\right) \sqrt{1-(1-X)\left(1-Y_{L}^{2}\right) / Y_{T}^{2}}} .
\end{gathered}
$$

Near the transition angle, the denominator of the term on the right of (41b) is small, giving: a larger damping term than that of the quasi-electromagnetic wave. At the transition angle, damping will be greater, the smaller the value of $V$. For very small values of $V$, corresponding to electron temperatures in the range $10^{3}{ }^{\circ} \mathrm{K}$ or less, the damping will probably be so great as to prevent observation of a quasi-acoustic wave at wave-normal angles near the transition angle in any part of the ionosphere.

\section{Conclusions}

From the analysis presented here it appears that quasi-acoustic waves can be propagated within the ionosphere at VLF frequencies provided the electron temperature is sufficiently high $\left(10^{4}-10^{5} \mathrm{~K}\right)$. These waves will be coupled with the propagating electromagnetic waves, the so-called "whistler" mode of magneto-ionic theory, near the critical angle between the wave normal and the direction of the geomagnetic field.

The effect of losses due to collisions between electrons and heavy particles is important, and in general the quasi-acoustic wave is subject to greater damping than is the electromagnetic wave. The damping of the quasi-acoustic wave increases with increasing electron density and decreases with increasing electron temperature. Damping prevents observation of the quasi-acoustic mode in the lower part of the ionosphere.

It also appears unlikely that waves propagating in a natural whistler mode which couples in the outer ionosphere to an acoustic wave will be able to be detected at the earth's surface, although this conjecture has not been confirmed by ray-tracing calculations. However, only waves which have wave normal directions near the critical angle with respect to the geomagnetic field will be appreciably coupled, and such waves may either be "lost," leaving the earth's field, or may be highly attenuated in the lower region of the ionosphere.

Acoustic waves at VLF may also be directly excited by radio transmitters located on earth satellites or by large-scale disturbances, such as nuclear explosions in the upper atmosphere. The possibility of excitation of such waves should be taken into account in the design of VLF satellite transmitting antennas, as the effect on the antenna impedance could be appreciable for certain orientations with respect to the geomagnetic field.

\section{References}

Cain, J. C., I. R. Shapiro, J. D. Stolarik, and J. P. Heppner (Sept. 1961), A note on whistlers observed above the ionosphere, J. Geophys. Res. 66, 2677-2680.

Cowling, T. G. (1945), The electrical conductivity of an ionized gas in a magnetic field with applications to the solar atmosphere and the ionosphere, Proc. Roy. Soc. $\mathbf{1 8 3}(\mathbf{A}), 453$. 
Helliwell, R. A., and M. G. Morgan (Feb. 1959), Atmospheric whistlers, Proc. IRE 47, 200-208.

Leiphart, J. P., R. W. Zeek, L. S. Bearce, and E. Toth (Jan. 1962), Penetration of the ionosphere by very-low frequency radio signals-Interim results of the Lofti I experiment, Proc. IRE 50, 6-17.

Oster, L. (Jan. 1960), Linearized theory of plasma oscillations, Rev. Mod. Phys. 32, 141-168.

Smith, R. L., R. A. Helliwell, and I. W. Yabroff (Mar. 1960), A theory of trapping of whistlers in field alined columns of enhanced ionization, J. Geophys. Res. 65, 815-823.

Storey, L. R. O. (July 1953), An investigation of whistling atmospherics, Phil. Trans. Roy. Soc. 246(A), 113 141.

Yabroff, I. (Sept. 1961), Computation of whistler ray paths, J. Res. NBS 65D (Radio Prop.), No. 5, 485-505.

(Paper 68D7-376) 